\title{
A COMPREHENSIVE STUDY OF OTITIS MEDIA WITH EFFUSION IN PAEDIATRIC AGE GROUP
}

\author{
M. Muniraju1, C. Shivani² \\ ${ }_{1}^{1}$ Associate Professor, Department of ENT, Dr. B. R. Ambedkar Medical College, Bengaluru. \\ 2Junior Resident, Department of ENT, Dr. B. R. Ambedkar Medical College, Bengaluru.
}

ABSTRACT

\section{BACKGROUND}

The objective of this study is to assess the incidence, various aetiological agents and early diagnosis and management of otitis media with effusion (OME).

\section{MATERIALS AND METHODS}

A total of 1800 paediatric age group patients aged between 5 to 15 years attending ENT OPD of Dr. B. R. Ambedkar Medical College and Hospital from October 2014 to October 2016 were screened, and 100 patients suffering from OME were included in the study after taking their consent. Patients with OME were assessed with the help of detailed history, clinical examination, diagnostic nasal endoscopy, relevant haematological, radiological, tuning fork tests and audiometric investigations.

\section{RESULTS}

The incidence of secretory otitis media was noted to be highest in the age group of 7-8 years with female preponderance. Bilateral hearing loss was seen in $90 \%$ of patients and unilateral hearing loss was present in $10 \%$ of patients. Symptoms of adenoid hypertrophy was noted in $98 \%$ patients. Tuning fork tests, audiometry, acoustic reflex tests and x-ray of soft tissue of nasopharynx lateral view showed correlation between adenoid hypertrophy and conductive hearing loss in development of OME. No significant improvement was noted following medical management. All patients underwent adenoidectomy and tympanostomy tube insertion, a statistically significant reduction in air conduction threshold was observed. Except in eight patients there is no significant reduction in air conduction threshold due to otorrhoea secondary to persistent perforation following tympanostomy tube insertion.

\section{CONCLUSION}

Bilateral hearing impairment, symptoms of adenoid hypertrophy, significant conductive hearing impairment ( $>20 \mathrm{~dB})$, abnormal findings on otoscopy, audiogram and acoustic reflex are present in more than three fourths of children presenting with OME. Tympanostomy tube insertion and adenoidectomy done in a single setting resulted in significant improvement in hearing without any major complications.

\section{KEYWORDS}

Serous Otitis Media, Secretory Otitis Media and Popularly Glue Ear.

HOW TO CITE THIS ARTICLE: Muniraju M, Shivani C. A comprehensive study of otitis media with effusion in paediatric age group. J. Evolution Med. Dent. Sci. 2017;6(17):1352-1356, DOI: 10.14260/Jemds/2017/294

\section{BACKGROUND \\ Otitis media with effusion is a common childhood otological condition. It is defined as an inflammation of middle ear with accumulation of fluid behind an intact tympanic membrane, without the signs and symptoms of acute infection. ${ }^{1}$ Persistent OME: Symptoms of OME, mainly hearing impairment persisting for more than 3 months. Diagnosis in children is often delayed as they cannot complain of hearing loss. Otitis media with effusion (OME) has a high prevalence in children and is responsible for most of the hearing losses in school going age (5-12 years) group. ${ }^{1}$ Most cases of OME are relatively asymptomatic with nearly 25\% discovered incidentally.}

Financial or Other, Competing Interest: None.

Submission 17-01-2017, Peer Review 14-02-2017,

Acceptance 20-02-2017, Published 27-02-2017.

Corresponding Author:

Dr. M. Muniraju,

Associate Professor,

Department of ENT

Dr. B. R. Ambedkar Medical College,

Kadugondanahalli, Shampura Main Road,

Bengaluru-560045,

Karnataka, India.

E-mail:drmuniraju@yahoo.in

DOI: $10.14260 /$ jemds $/ 2017 / 294$

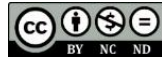

Despite this apparent absence of symptoms, the potential impact on hearing, speech, language and cognition highlights the need for timely intervention. ${ }^{2}$ The aetiologies of OME are very diverse and include viral and bacterial infection, Eustachian tube dysfunction and excessive exudate production due to allergy and chronic inflammation. ${ }^{3}$ Appropriate evaluation, early and effective treatment is of utmost importance to prevent morbidity and sequelae. In persistent OME with hearing impairment, the current standard of care is conservative treatment and if necessary insertion of tympanostomy tube as the first step followed by adenoidectomy in resistant cases or when symptoms of adenoid hypertrophy are prominent.

\section{MATERIALS AND METHODS}

The present study "A comprehensive study of otitis media with effusion in paediatric age group" has been carried out in the Department of ENT, Dr. B. R. Ambedkar Medical College, Bengaluru over a period of 2 years. During this period, 1800 paediatric age group patients aged between 5 to 15 years were evaluated by detailed ear, nose and throat examination and 100 patients with otitis media with effusion having potential impact on hearing and development were included in the present study after excluding patients with mixed or 
sensorineural hearing loss, ear discharge, cleft lip or cleft palate, patients with speech and laryngeal maldevelopment, nasopharyngeal tumour, unilateral otitis media and otosclerosis. Hearing threshold of both ears were determined by pure tone audiometry. The type of tympanogram and stapedial reflex was recorded. Tuning fork tests were performed wherever possible. Patients underwent routine investigations and other relevant investigations like X-ray of nasopharynx as per the requirement after taking their consent. Most of these patients had taken medical line of treatment for at least 3 months before coming to us. These patients were posted for tympanostomy tube insertion and adenoidectomy without any delay. Other fresh cases of secretory otitis media were given nasal decongestants, antihistamine and $\mathrm{N}$-acetylcysteine for a duration of 3 months and planned for tympanostomy tube insertion and adenoidectomy on the affected side. They were followed up at weekly intervals for a month, monthly intervals for a period of 6 months and after 1 year. They were regularly assessed for complications, subjective improvement in hearing, otorrhoea and extrusion of tympanostomy tube.

\section{RESULTS}

In this study of 100 cases, incidence of secretory otitis media was noted to be highest in the age group of 7-8 years and there were 40 male cases and 60 female cases. Male to female ratio of 2:3 showed female preponderance in this study. Incidence of bilateral hearing loss was seen in $90 \%$ of patients and unilateral hearing loss was present in $10 \%$ of patients (right - 8\% and left - 2\%). 98\% patients have symptoms of adenoid hypertrophy - mouth breathing, snoring, speech hyponasality. Aetiology of OME is multifactorial and many different factors are implicated in the pathophysiology of the disease. Hence the main responsible factors for development of OME in remaining $2 \%$ could be because of recurrent URTI, socioeconomic class, passive smoking and allergy, etc. The evidence of OME on otoscopic examination was observed in at least one or both the ears of patients in this study. Tympanic membrane abnormality suggestive of OME was bilateral in $90 \%$ patients. Abnormality of tympanic membrane mobility (absent or restricted) was documented in at least one ear in all hundred patients. 92 patients had abnormal mobility of tympanic membrane in left ear and 98 patients had abnormal mobility of tympanic membrane on right side. There was a good correlation between patients presenting with hearing impairment and otoscopic abnormalities.

Tuning Fork test showed 76 had conductive hearing loss on left side and 82 had conductive hearing loss on right side. However, in 16 patients TFT was inconclusive as these children were not cooperative.

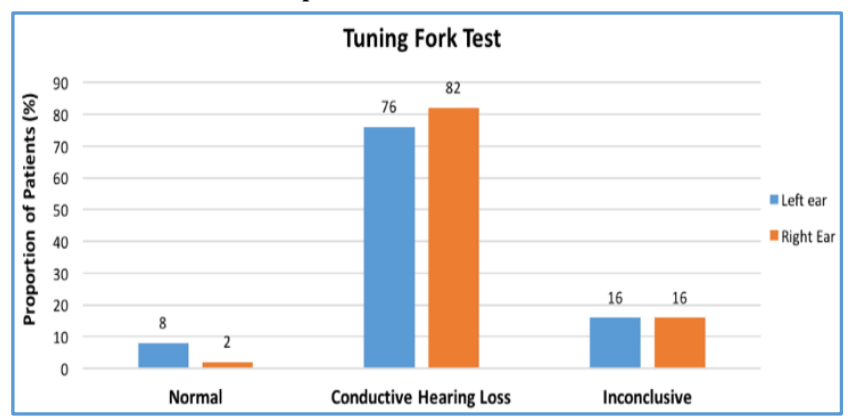

Graph 1. Tuning Fork Test
Type B pattern on tympanometry suggestive of little or no change in compliance of tympanic membrane and characteristic of OME was observed in 88 patients in left ear and 98 patients in right ear. Type $C$ pattern on tympanometry suggestive of negative middle ear pressure and characteristics of early OME was seen in 4 patients in left ear.

The severity of adenoid hypertrophy was assessed by Xray of soft tissue of nasopharynx lateral view. Twenty four patients have palatal airway $<5 \mathrm{~mm}$ and sixty four patients have palatal airway $6-10 \mathrm{~mm}$ that is 88 patients have some palatal narrowing at the time of presentation. On analysing the relationship between palatal airway and age of patients, it was observed that children in the age group of 5 to 8 years have statistically significant airway narrowing (adenoid hypertrophy) than children in the age group of 9-12 years.

\begin{tabular}{|c|c|c|c|}
\hline $\begin{array}{l}\text { Age in } \\
\text { Years }\end{array}$ & $\begin{array}{c}\text { Palatal } \\
\text { Airway } \\
<5 \mathrm{~mm} \\
\text { Number of } \\
\text { Patients (\%) }\end{array}$ & \begin{tabular}{|c|} 
Palatal \\
Airway \\
6-10 mm \\
Number of \\
Patients (\%)
\end{tabular} & $\begin{array}{c}\text { Palatal } \\
\text { Airway } \\
>10 \mathbf{~ m m} \\
\text { Number of } \\
\text { Patients (\%) }\end{array}$ \\
\hline $5-6$ & $8(8 \%)$ & $6(6 \%)$ & $2(2 \%)$ \\
\hline $7-8$ & $10(10 \%)$ & $24(24 \%)$ & $6(6 \%)$ \\
\hline $9-10$ & $2(2 \%)$ & $18(18 \%)$ & $2(2 \%)$ \\
\hline 11-12 & $4(4 \%)$ & $16(16 \%)$ & $2(2 \%)$ \\
\hline $\begin{array}{c}\text { Total } \\
(\mathrm{n}=\mathbf{5 0} \\
\text { Patients })\end{array}$ & $24(24 \%)$ & $64(64 \%)$ & $12(12 \%)$ \\
\hline
\end{tabular}

A baseline air conduction threshold at pure tones $0.5,1,2$ and $4 \mathrm{KHz}$ was determined prior to surgery. The mean air conduction threshold on audiometry was $41.97 \mathrm{dBHL}$ (Standard deviation - 8.23) and 38.98 dBHL (Standard deviation -10.97) on right and left side respectively.

In this present study out of 100 patients, few patients had taken antihistamines, nasal decongestants and mucolytics for a period of 3 months before approaching us. Hence, these patients were taken up for surgery immediately. Other patients who had not taken any medical treatment were given antihistamines, nasal decongestants, mucolytics for 3 months. PTA and impedance repeated after 3 months to confirm the presence of middle ear effusion.

All patients underwent tympanostomy tube insertion and adenoidectomy. Tympanostomy tube insertion was bilateral in 90 patients and unilateral in 10 patients. No intraoperative or postoperative complications were observed. All patients were discharged on first postoperative day. During the followup 12 patients complained of earache in the first 2 weeks following surgery and were symptomatically treated. Eight patients on regular followup at the end of 1 year showed no improvement in hearing due to otorrhoea secondary to persistent perforation.

The effect of tympanostomy tube placement along with adenoidectomy on hearing was assessed by considering air conduction thresholds as continuous variables as well as categorising them arbitrarily into 3 categories. On analysing serial air conduction thresholds at baseline, 2 months, 6 months and 1 year following surgical intervention, a statistically significant reduction in air conduction threshold was observed (Repeated measures ANOVA, $\mathrm{p}=<0.001$ ). Except in eight patients, there was no significant reduction in 
air conduction threshold due to otorrhoea secondary to persistent perforation. On intra-group comparison of air conduction thresholds, it was noted that significant reduction in air conduction threshold occurred postoperatively for the first 6 months. No significant improvement observed on PTA after 1 year duration.

\begin{tabular}{|c|c|c|c|}
\hline AC dBHL & Pre-0p & $\begin{array}{c}\text { Post-Op } \\
\text { 2nd }^{\text {Month }}\end{array}$ & $\begin{array}{c}\text { Post-Op } \\
6^{\text {th }} \text { Month }\end{array}$ \\
\hline $\begin{array}{c}\text { Right ear AC dB } \\
\text { HL (Mean } \pm \text { SD) }\end{array}$ & $41.97 \pm 8.23$ & $29.43 \pm 8.22$ & $20.21 \pm 8.78$ \\
\hline $\begin{array}{c}\text { Left ear AC dB } \\
\text { HL (Mean } \pm \text { SD) }\end{array}$ & $38.98 \pm 10.97$ & $27.09 \pm 7.29$ & $18.02 \pm 4.99$ \\
\hline
\end{tabular}

Table 2. Effect of Tympanostomy and Adenoidectomy on Air Conduction (AC) Threshold on Mean Pure Tone Audiometry at 0.5, 1, 2, $4 \mathrm{KHz}$

On categorising air conduction thresholds into 3 groups, the effect of tympanostomy tube insertion and adenoidectomy on hearing impairment was analysed. Sixty eight out of 78 patients with significant hearing loss $(>20$ dBHL) in left ear and 72 out of 82 patients in right ear respectively returned to normal hearing thresholds $<<20$ dBHL) following surgery.

\section{DISCUSSION}

OME is a middle ear disease characterised by the presence of fluid in the middle ear resulting in decreased mobility of tympanic membrane and a conductive type of hearing loss. ${ }^{4}$ It is one of the most common health problems seen in children, leading cause of healthcare visits worldwide, and when inadequately treated or left untreated, it may lead to sequelae and complications like adhesive otitis media, retraction pocket and impairment in development of speech and language. The aetiology of $\mathrm{OME}$ is multifactorial. 5 Unfortunately, early diagnosis is not always possible since the disease is insidious and seen usually in small children. ${ }^{5}$ Therefore, it is essential to demonstrate the risk factors and prevalence of OME clearly for both disease prevention and for establishing a proper treatment plan.

There is a considerable variation of prevalence rates among the worldwide OME reports ranging from 1.3-61\%, depending on the methods used, race and population. However, irrespective of methods used, all studies conclude that age is one of the most important risk factor for OME. In this comprehensive study, 100 children with OME and persistent hearing impairment are recruited and outcome following surgical treatment is studied. The incidence of secretory otitis media is highest in the age group of 7-8 years. The mean (SD) age of patients in this study is 8.6 (1.9) years. There is no difference in mean gestational age between males and females. Similar results are seen in the study conducted by Reddy6 in 1998.

The influence of gender on OME is also quite controversial. There is a female preponderance in this study. But study conducted by Reddy 6 showed boys are more frequently affected than girls, which may be a reflection of anatomical or growth differences or the overall male predominance for childhood infection. Boys also have less well-pneumatised air cell system which may be a consequence of tubal dysfunction.
In our study, most of the patients presented with chief complaint of hearing impairment which is noted by parents or teachers more often. In the present study, $44 \%$ patients presented with hearing impairment persisting for last 7 to 12 months. Multiple trials with unproven conservative therapies and delay in referral by primary physicians to otolaryngologist could be responsible for prolonged duration of hearing impairment noted in this study. Symptoms of adenoid hypertrophy - Mouth breathing is observed in 98 patients, Snoring-96 patients, Speech hyponasality-64 patients. Hard of hearing is the main complain in the study conducted by Reddy. ${ }^{6}$

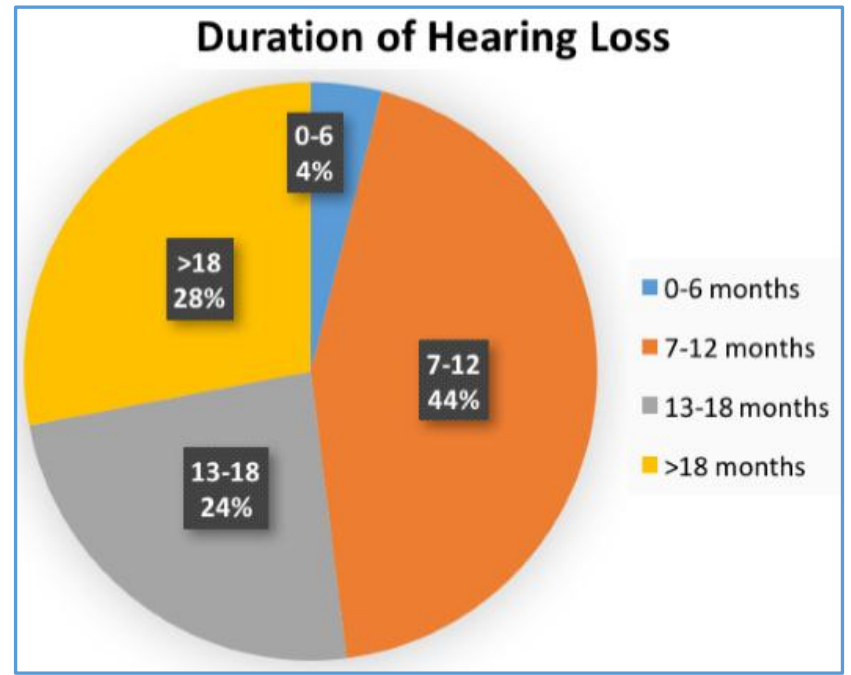

Graph 2. Duration of Hearing Loss at Presentation

AAP guideline recommends using pneumatic otoscopy as a primary method for diagnosing OME and tympanometry can be used to confirm the diagnosis". ${ }^{7}$ Acoustic reflexometry and non-pneumatic otoscopy are not advised for primary diagnosis because of the low reliability. ${ }^{8}$ Main features in diagnostic assessment of tympanic membrane in OME used in this study were classified by Kaleida and Fireman into colour, mobility, translucency, fluid appearance and retraction. Tympanic membrane findings that are suggestive of OME are colour-pearly grey, Opacification of tympanic membrane, fluid level, decreased mobility and retracted position. ${ }^{9}$ In our study, tympanic membrane abnormality suggestive of OME is bilateral in 90 patients. Majority of the patients showed dull and retracted tympanic membrane on otoscopic examination as the most common sign. This is seen in mucoid type of secretory otitis media. This is noted in $58 \%$ of cases in both right and left ear. Bulged tympanic membrane is noted in $26 \%$ of cases in left ear and $28 \%$ of cases in right ear. Air bubbles are present in $4 \%$ of cases in left ear and $8 \%$ of cases in right ear. This is generally seen in case of serous type of secretory otitis media. The bubbles are usually not seen unless the patient has positive pressure applied to closed nasopharynx. Thin and retracted membrane is noted in $4 \%$ of cases in left ear and $2 \%$ of cases in right ear. This implies a favourable prognostic sign. In the study conducted by Reddy ${ }^{6}$ (1998), dull and retracted tympanic membrane is the common finding. Abnormality of tympanic membrane mobility (Absent or restricted) is documented in at least one ear in all hundred patients. 
The tympanometry test is another method that can be used as a screening tool which is more objective.10 Tympanograms were assessed according to the original classification by Jerger, as modified by Orchik et al and Zielhuis et al. Peaked tympanograms were classified to be type A when tympanometric peak pressure was over -100 daPa, type $\mathrm{C} 1$ when pressure was between -100 and -200 $\mathrm{daPa}$, type $\mathrm{C} 2$ when pressure was lower than $-200 \mathrm{daPa}$ and type Cs when the tympanogram was low peaked and wide (Peak compliance less $<0.2$ mmho, width over $300 \mathrm{daPa}$ ). A flat tympanogram was classified to be type B. It has been demonstrated that $85-100 \%$ of children with OME will have type B tympanogram, $20-50 \%$ type C2, 15\% type C1 and less than $5 \%$ type A. ${ }^{10}$ Thus, in this study, type B and C tympanogram were taken to indicate the presence of OME. Another diagnostic tool for detecting the presence of OME in this study is the absence of acoustic reflex. The absence of middle ear reflex have been shown to indicate the presence of OME with a sensitivity of $95-97 \%$ but low specificity of $25-$ $50 \% .11,12$ With the combination of all the three methods the sensitivity and specificity of presence of OME can be brought up to $90-97 \% .^{12}$ Therefore, in this study, we took into consideration all the three methods, and the presence of OME is considered when there is abnormal otoscopic finding by ENT surgeon, Type B tympanogram and absence of ipsilateral acoustic reflex in both ears. There is a good correlation between patients presenting with hearing impairment and otoscopic abnormalities.

Conductive hearing loss in OME results from the attenuation of sound signals reaching the inner ear'13. It is common sequelae of OME due to the fluid and changes of tympanic membrane due to inflammation. Scarring of tympanic membrane or adhesive retraction of the ear drum to the ossicles can cause conductive hearing loss. Otitis media with effusion usually results in conductive hearing loss of 20 - $30 \mathrm{~dB}$ and degree of loss can vary depending on character and quality of effusion. Fria's et al reported an average air conduction threshold of $27 \mathrm{~dB}$ in 540 children aged 2-12 years old with OME. ${ }^{13}$ The average hearing level in our study was $41.97 \mathrm{dBHL}$ (Standard deviation - 8.23) and $38.98 \mathrm{dBHL}$ (Standard deviation-10.97) on right and left side respectively. The highest hearing loss in this study could be due to ignorance and late reporting of patients in this area.

Many management strategies have been advocated for glue ear. The purpose of treatment is to remove the mucous secretion, prevent its recurrence and re-establish the permeability of the eustachian tube. Antibiotics have been used because up to $50 \%$ of chronic middle ear effusion are culture positive. ${ }^{14}$, Antimicrobial agents may therefore induce resolution of the effusion. Usually amoxicillin and trimethoprim-sulfisoxazole have been found effective in eradicating middle ear effusion. Antihistamine-Decongestant combinations are not helpful in non-allergic children with middle ear effusion but uncertain results are seen in allergic children. Controversy also exists regarding the administration of steroids. Oral antiallergic drugs may prove effective in patients with otitis media with effusion and allergic rhinitis. ${ }^{15}$ Mucolytic agents have been used but their efficacy has not been established. 16

Eustachian tube inflation (Valsalva manoeuvre or Politzerisation) is rarely successful in chronic middle ear effusion, so surgery should be considered. Surgical therapy does not cure patients with secretory otitis media; only time, growth and development can. But it does substantially reduce morbidity when medical therapy has failed. Myringotomy, tympanotomy and ventilation tube insertion with or without adenoidectomy have been found to reduce conductive hearing loss secondary to middle ear effusion. Hearing depends on middle ear status. When no effusion is present, hearing is within normal limits, but when effusion occurred hearing threshold is elevated. Similarly, the air bone gap is proportional to the amount of fluid in the middle ear.

In the present study, surgery is performed in children with pure tone air conduction average of $>20 \mathrm{~dB}$, since hearing loss places children at risk for language and learning problems.

In the present study, we performed adenoidectomy and tympanostomy in a single setting. This practice is adopted for the following reasons-

- Adenoidectomy has been found to potentially benefit patients with OME even in the absence of adenoid hypertrophy. ${ }^{17}$

- Adenoidectomy ensures removal of potential source for persistent OME.

- Patients included in this study have OME with persistent hearing impairment and chances of spontaneous resolution in this setting would be extremely low.

- Anticipated reluctance of patients for constant followup and readmission for second surgery.

- Adenoidectomy along with tympanostomy tube insertion, when compared to tympanostomy tube insertion alone, required fewer re-insertions to maintain adequate subjective hearing thresholds in the treated ears. ${ }^{18}$

In the present study, Teflon tympanostomy tubes were used. The insertion was done in the anteroinferior quadrant of the tympanic membrane as it is more physiological. ${ }^{19}$

Adenoidectomy and Tympanostomy tube insertions were done in a single setting in all patients. ${ }^{20}$

All patients were available for followup. Pure tone audiometry (PTA) is recorded at $2^{\text {nd }}$ month, $6^{\text {th }}$ month and after 1 year postoperatively. Two, six months and 1 year time periods were chosen to ensure good patient compliance and also to ensure sufficient time is available to document all important clinical outcomes. However, Maw AR et al followed up patients for a period of 12 months following surgery.

Improvement in air conduction thresholds to $<20 \mathrm{~dB}$ was noted in 72 patients in the right ear and 68 patients in the left ear at the end of six months following surgery.

Except in eight patients there is no significant reduction in air conduction threshold due to otorrhoea secondary to persistent perforation. On intragroup comparison of air conduction thresholds, it was noted that significant reduction in air conduction threshold occurred postoperatively for the first 6 months. No significant improvement observed on PTA after 1 year duration.

\section{CONCLUSION}

Otitis media continues to affect millions of children around the world annually with attendant complications. High index of suspicion is required to ensure prompt diagnosis and treatment in order to drastically reduce the incidence of 
sequelae. Bilateral hearing impairment, symptoms of adenoid hypertrophy, significant hearing impairment $(>20 \mathrm{~dB})$, abnormal findings on otoscopy, acoustic reflex and tympanometry are present in more than three fourths of children presenting with OME and hearing impairment. Tympanostomy tube insertion and adenoidectomy done in a single setting resulted in significant improvement in hearing impairment without any major complications.

\section{REFERENCES}

[1] Rosenfeld RM. Comprehensive management of otitis media with effusion. Otolaryngol Clin North Am 1994;27(3):443-55.

[2] Motamed M, Thorne S, Narula A. Treatment of otitis media with effusion in children with mucopolysaccharidoses. International Journal of pediatric otorhinolaryngology 2000;53(2):121-4.

[3] Choung YH, Shin YR, Choi SJ, et al. Management for the children with otitis media with effusion in the tertiary hospital. Clin Exp Otorhinolaryngol 2008;1(4):201-5.

[4] Apostolopoulos K, Xenelis J, Tzagaroulakis A, et al. The point prevalence of otitis media with effusion among school children in Greece. Int J Pediatr Otorhinolaryngol 1998;44(3):207-14.

[5] Gates GA. Acute otitis media and otitis media with effusion. In: Cummings CW (edr). Otolaryngology, head \& neck surgery. 3rd edn. St Louis: Mosby 1998.

[6] Reddy VG. Secretory otitis media. Indian journal of otology 1998;4(4):157-60.

[7] American Academy of Family Physicians; American Academy of Otolaryngology Head and Neck Surgery; American Academy of Pediatrics Subcommittee on Otitis Media With Effusion. Otitis media with effusion. Pediatrics 2004;113(5):1412-29.

[8] Fria TJ, Cantekin EI, Eichler JA. Hearing acuity of children with otitis media with effusion. Arch otolaryngology 1985;111(1):10-6.

[9] Kaleida PH, Stool SE. Assessment of otoscopist's accuracy regarding middle-ear effusion. Otoscopic validation. Am J Dis Child 1992;146(4):433-5.
[10] Saim A, Saim L, Saim S, et al. Prevalence of otitis media with effusion amongst pre-school children in Malaysia. Int J Pediatr Otorhinolaryngol 1997;41(1):21-8.

[11] Nozza RJ, Bluestone CD, Kardatzke D, et al. Identification of middle ear effusion by aural acoustic admittance and otoscopy. Ear earing 1994;15(4):31023.

[12] Cantekin EI, Bluestone CD, Fria TJ, et al. Identification of otitis media with effusion in children. Ann Otol Rhinol Laryngol Suppl 1980;89(3 Pt 2):190-5.

[13] Zielhuis GA, Gerritsen AA, Gorissen WH, et al. Hearing deficits at school age; the predictive value of otitis media in infants. Int $\mathrm{J}$ Pediatr Otorhinolaryngol 1998;44(3):227-34.

[14] American Academy of Pediatrics Subcommittee on Management of Acute Otitis Media. Diagnosis and management of acute otitis media. Pediatrics 2004;113(5):1451-65.

[15] Suzuki M, Kawauchi H, Mogi G. Clinical efficacy of an antiallergic drug on otitis media with effusion in association with allergic rhinitis. Auris Nasus Larynx 1999;26(2):123-9.

[16] Paparella MM. Middle ear effusions: definitions and terminology. Ann otol Rhinol Laryngol 1976;85(2 Suppl 25 Pt 2):8-11.

[17] Maw RA. Otitis media with effusion. In: Adams DA, Cinnamond MJ, (edr). Scott-Brown's otolaryngology. $6^{\text {th }}$ edn. Vol 6. Butterworth-Heinemanl 1997:1-23.

[18] Tuli BS, Singh B, Parmar TL. Evaluation of tympanostomy tubes in middle ear affections. Indian Journal of Otolaryngology 2001;53(3):217-20.

[19] Dingle AF, Flood LM, Kumar BU, et al. Tympanosclerosis and mini grommets: the relevance of grommet design. J Laryngol Otol 1995;109(10):9225.

[20] Morris MS. Tympanostomy tubes: types, indications, techniques, and complications. Otolaryngol Clin North Am 1999;32(3):385-90. 